\title{
Bone Specific Alkaline Phosphatase
}

National Cancer Institute

\section{Source}

National Cancer Institute. Bone Specific Alkaline Phosphatase. NCI Thesaurus. Code C113515.

An isoform of alkaline phosphatase found on the surface of osteoblasts. The level of this enzyme in a patient's serum is proportional to the amount of bone formation. This marker is used for monitoring the efficacy of both bone formation therapies and antiresorptive therapies. 